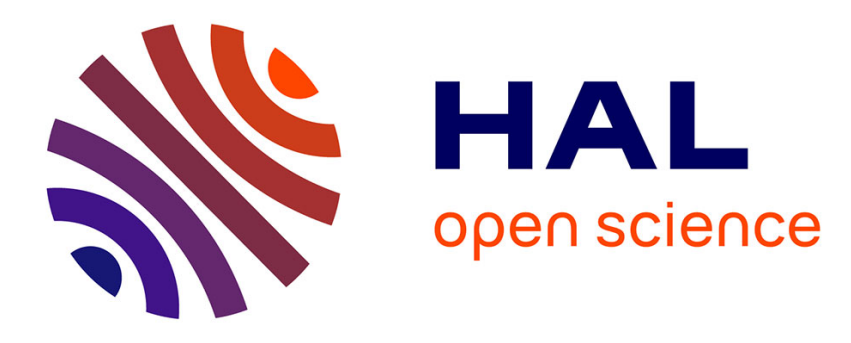

\title{
Decidable Approximations of Sets of Descendants and Sets of Normal forms
}

\author{
Thomas Genet
}

\section{To cite this version:}

Thomas Genet. Decidable Approximations of Sets of Descendants and Sets of Normal forms. 9th Conference on Rewriting Techniques and Applications, 1998, Tsukuba, Japan, pp.151-165. inria00098700

\section{HAL Id: inria-00098700 \\ https://hal.inria.fr/inria-00098700}

Submitted on 26 Sep 2006

HAL is a multi-disciplinary open access archive for the deposit and dissemination of scientific research documents, whether they are published or not. The documents may come from teaching and research institutions in France or abroad, or from public or private research centers.
L'archive ouverte pluridisciplinaire HAL, est destinée au dépôt et à la diffusion de documents scientifiques de niveau recherche, publiés ou non, émanant des établissements d'enseignement et de recherche français ou étrangers, des laboratoires publics ou privés. 


\title{
Decidable Approximations of Sets of Descendants and Sets of Normal Forms
}

\author{
Thomas Genet \\ INRIA Lorraine \& CRIN CNRS - BP 101 \\ 54602 Villers-lès-Nancy Cedex FRANCE \\ Phone: (+33) 3-83-59-30-18 - Fax: (+33) 3-83-27-83-19 \\ E-mail: Thomas.Genet@loria.fr \\ http://www.loria.fr/equipe/protheo.html/
}

\begin{abstract}
We present here decidable approximations of sets of descendants and sets of normal forms of Term Rewriting Systems, based on specific tree automata techniques. In the context of rewriting logic, a Term Rewriting System is a program, and a normal form is a result of the program. Thus, approximations of sets of descendants and sets of normal forms provide tools for analysing a few properties of programs: we show how to compute a superset of results, to prove the sufficient completeness property, or to find a criterion for proving termination under a specific strategy, the sequential reduction strategy. The main technical contribution of the paper is the construction of an approximation automaton which recognises a superset of the set of normal forms of terms in a set $E$, w.r.t. a Term Rewriting System $\mathcal{R}$.
\end{abstract}

\section{Introduction}

In the context of the programming language such as ELAN [18], a Term Rewriting System (TRS for short) is a program. We propose here to use tree automata techniques for proving various properties on TRSs and thus on programs. For a given TRS $\mathcal{R}$ and a set of terms $E$, these proofs are based on the computation of approximations of the set of $\mathcal{R}$-descendants of $E$ and the set of $\mathcal{R}$-normal forms of $E$. For that, we build an approximation automaton which recognises a superset of the set of $\mathcal{R}$-descendants and $\mathcal{R}$-normal forms of terms in $E$. Considering $\mathcal{R}$ as a program and $E$ as the set of possible inputs of the program, the set of $\mathcal{R}$ descendants of $E$ represents all intermediate results of the program at every step of its execution on the given set of possible inputs. The set of $\mathcal{R}$-normal forms of $E$ represents the set of all possible results obtained by executing the program $\mathcal{R}$ on the set of possible given inputs $E$, when the program stops. Thanks to those two sets, we show how to prove sufficient completeness of a program on a set of possible initial inputs, how to achieve some reachability testing on a program, and how to prove termination of a program represented by a TRS and a strategy of application of rewrite rules called sequential reduction strategy.

In Section 1, we recall basic definitions of terms, term rewriting systems, and tree automata. In Section 2, we briefly present sufficient completeness, reachability testing and termination proof under the sequential reduction strategy. Then, 
in Section 3, we recall some undecidability results on the set of descendants and the set of normal forms motivating our approach by approximation. We also detail the approximation construction which is based on specific matching and rewriting techniques on tree automata, schematising matching and rewriting on sets of terms. Feasibility of the approximation construction and its appropriateness for our purpose is shown in Section 4 on some examples. Some automatic proofs achieved by our prototype are also presented in Section 4. Finally we conclude on this work in Section 5.

\section{Preliminaries}

We now introduce some notations and basic definitions. Comprehensive surveys can be found in [9] for term rewriting systems, in $[11,4]$ for tree automata and tree language theory, and in [15] for connections between regular tree languages and term rewriting systems.

\section{Terms, Substitutions, Rewriting systems}

Let $\mathcal{F}$ be a finite set of symbols associated with an arity function denoted by ar $: \mathcal{F} \mapsto \mathbb{N}, \mathcal{X}$ be a countable set of variables, $\mathcal{T}(\mathcal{F}, \mathcal{X})$ the set of terms, and $\mathcal{T}(\mathcal{F})$ the set of ground terms (terms without variables). Positions in a term are represented as sequences of integers. The set of positions in a term $t$, denoted by $\mathcal{P} o s(t)$, is ordered by lexicographic ordering $\prec$. The empty sequence $\epsilon$ denotes the top-most position. $\operatorname{Root}(t)$ denotes the symbol at position $\epsilon$ in $t$. For any term $s \in \mathcal{T}(\mathcal{F}, \mathcal{X})$, we denote by $\operatorname{Pos}_{\mathcal{F}}(s)$ the set of functional positions in $s$, i.e. $\left\{p \in \mathcal{P}\right.$ os $(s) \mid p \neq \epsilon$ and $\left.\operatorname{Root}\left(\left.s\right|_{p}\right) \in \mathcal{F}\right\}$. If $p \in \mathcal{P}$ os $(t)$, then $\left.t\right|_{p}$ denotes the subterm of $t$ at position $p$ and $t[s]_{p}$ denotes the term obtained by replacement of the subterm $\left.t\right|_{p}$ at position $p$ by the term $s$. A ground context is a term of $\mathcal{T}(\mathcal{F} \cup\{\square\})$ with only one occurrence of $\square$, where $\square$ is a special constant not occurring in $\mathcal{F}$. For any term $t \in \mathcal{T}(\mathcal{F}), C[t]$ denotes the term obtained after replacement of $\square$ by $t$ in the ground context $C[]$. The set of variables of a term $t$ is denoted by $\operatorname{Var}(t)$. A term is linear if any variable of $\mathcal{V} a r(t)$ has exactly one occurrence in $t$. A substitution is a mapping $\sigma$ from $\mathcal{X}$ into $\mathcal{T}(\mathcal{F}, \mathcal{X})$, which can uniquely be extended to an endomorphism of $\mathcal{T}(\mathcal{F}, \mathcal{X})$. Its domain $\operatorname{Dom}(\sigma)$ is $\{x \in X \mid x \sigma \neq x\}$.

A term rewriting system $\mathcal{R}$ is a set of rewrite rules $l \rightarrow r$, where $l, r \in$ $\mathcal{T}(\mathcal{F}, \mathcal{X}), l \notin \mathcal{X}$, and $\mathcal{V}$ ar $(l) \supseteq \mathcal{V}$ ar $(r)$. A rewrite rule $l \rightarrow r$ is left-linear (resp. right-linear) if the left-hand side (resp. right-hand side) of the rule is linear. A rule is linear if it is both left and right-linear. A TRS $\mathcal{R}$ is linear (resp. leftlinear, right-linear) if every rewrite rule $l \rightarrow r$ of $\mathcal{R}$ is linear (resp. left-linear, right-linear).

The relation $\rightarrow_{\mathcal{R}}$ induced by $\mathcal{R}$ is defined as follows: for any $s, t \in \mathcal{T}(\mathcal{F}, \mathcal{X})$, $s \rightarrow_{\mathcal{R}} t$ if there exist a rule $l \rightarrow r$ in $\mathcal{R}$, a position $p \in \mathcal{P} o s(s)$ and a substitution $\sigma$ such that $l \sigma=\left.s\right|_{p}$ and $t=s[r \sigma]_{p}$. The transitive (resp. reflexive transitive) closure of $\rightarrow_{\mathcal{R}}$ is denoted by $\rightarrow_{\mathcal{R}}^{+}$(resp. $\rightarrow_{\mathcal{R}}^{*}$ ). A term $s$ is reducible by $\mathcal{R}$ if there exists $t$ s.t. $s \rightarrow_{\mathcal{R}} t$. 
A term $s$ is in $\mathcal{R}$-normal form (or is $\mathcal{R}$-irreducible) if $s$ is not reducible by $\mathcal{R}$. A term $s$ has a normal form if there exists a term $t$ in $\mathcal{R}$-normal form s.t. $s \rightarrow_{\mathcal{R}}^{*} t$. The set of all ground terms in $\mathcal{R}$-normal form is denoted by $\operatorname{IRR}(\mathcal{R})$, and $s \rightarrow_{\mathcal{R}}^{*} t$ with $t \in I R R(\mathcal{R})$ is denoted by $s \rightarrow_{\mathcal{R}}^{!} t$. The set of $\mathcal{R}$-descendants of a set of ground terms $E$ is denoted by $\mathcal{R}^{*}(E)$ and $\mathcal{R}^{*}(E)=\{t \in \mathcal{T}(\mathcal{F}) \mid \exists s \in$ $E$ s.t. $\left.s \rightarrow_{\mathcal{R}}^{*} t\right\}$. The set of ground $\mathcal{R}$-normal forms of $E$ is denoted by $\mathcal{R}^{!}(E)$ and $\mathcal{R}^{!}(E)=\left\{t \in \mathcal{T}(\mathcal{F}) \mid \exists s \in E\right.$ s.t. $\left.s \rightarrow_{\mathcal{R}}^{!} t\right\}$. Moreover, $\mathcal{R}^{!}(E)=\mathcal{R}^{*}(E) \cap I R R(\mathcal{R})$.

A rewriting system $\mathcal{R}$ is (1) terminating or strongly normalising if there exists no infinite derivation $s_{1} \rightarrow_{\mathcal{R}} s_{2} \rightarrow_{\mathcal{R}} \ldots$ where $s_{1}, s_{2}, \ldots \in \mathcal{T}(\mathcal{F}, \mathcal{X}),(2)$ weakly normalising (WN for short) if every $s$ of $\mathcal{T}(\mathcal{F}, \mathcal{X}$ ) has a normal form, (3) weakly normalising on $E \subseteq \mathcal{T}(\mathcal{F}, \mathcal{X}$ ) (WN on $E$ ) if every $s \in E$ has a normal form.

The set of function symbols $\mathcal{F}$ occurring in a TRS $\mathcal{R}$ can be partitioned into the set of defined symbols $\mathcal{D}=\{\operatorname{Root}(l) \mid l \rightarrow r \in \mathcal{R}\}$ and the set of constructors $\mathcal{C}=\mathcal{F} \backslash \mathcal{D}$. A constructor term, is a ground term with no defined symbol. The set of constructor terms is denoted by $\mathcal{T}(\mathcal{C})$. Let $\mathcal{R}_{1}$ and $\mathcal{R}_{2}$ be TRSs with respective sets of symbols $\mathcal{F}_{1}$ and $\mathcal{F}_{2}$, respective sets of defined symbols $\mathcal{D}_{1}$ and $\mathcal{D}_{2}$, and respective sets of constructors $\mathcal{C}_{1}$ and $\mathcal{C}_{2}$. TRSs $\mathcal{R}_{1}$ and $\mathcal{R}_{2}$ are hierarchical if $\mathcal{F}_{2} \cap \mathcal{D}_{1}=\emptyset$ and $\mathcal{R}_{1} \subset \mathcal{T}\left(\mathcal{F}_{1} \backslash \mathcal{D}_{2}, \mathcal{X}\right) \times \mathcal{T}\left(\mathcal{F}_{1}, \mathcal{X}\right)$.

\section{Automata, Regular Tree Languages}

Let $\mathcal{Q}$ be a finite set of symbols, with arity 0 , called states. $\mathcal{T}(\mathcal{F} \cup \mathcal{Q})$ is called the set of configurations. A transition is a rewrite rule $c \rightarrow q$, where $c \in \mathcal{T}(\mathcal{F} \cup \mathcal{Q})$ and $q \in \mathcal{Q}$. A normalised transition is a transition $c \rightarrow q$ where $c=f\left(q_{1}, \ldots, q_{n}\right)$, $f \in \mathcal{F}, \operatorname{ar}(f)=n$, and $q_{1}, \ldots, q_{n} \in \mathcal{Q}$. A bottom-up finite tree automaton (tree automaton for short) is a quadruple $A=\left\langle\mathcal{F}, \mathcal{Q}, \mathcal{Q}_{f}, \Delta\right\rangle$, where $\mathcal{Q}_{f} \subseteq \mathcal{Q}$ and $\Delta$ is a set of normalised transitions. The rewriting relation induced by $\Delta$ is denoted by $\rightarrow_{\Delta}$. The tree language recognised by $A$ is $\mathcal{L}(A)=\{t \in \mathcal{T}(\mathcal{F}) \mid \exists q \in$ $\mathcal{Q}_{f}$ s.t. $\left.t \rightarrow_{\Delta}^{*} q\right\}$. For a given $q \in \mathcal{Q}$, the tree language recognised by $A$ and $q$ is $\mathcal{L}(A, q)=\left\{t \in \mathcal{T}(\mathcal{F}) \mid t \rightarrow_{\Delta}^{*} q\right\}$. A tree language (or a set of terms) $E$ is regular if there exists a bottom-up tree automaton $A$ such that $\mathcal{L}(A)=E$. The class of regular tree language is closed under boolean operations $\cup, \cap, \backslash$, and inclusion is decidable. A $\mathcal{Q}$-substitution is a substitution $\sigma$ s.t. $\forall x \in \mathcal{D} o m(\sigma), x \sigma \in \mathcal{Q}$. Let $\Sigma(\mathcal{Q}, \mathcal{X})$ be the set of $\mathcal{Q}$-substitutions. For every transition, there exists an equivalent set of normalised transitions. Normalisation consists in decomposing a transition $s \rightarrow q$, into a set $\operatorname{Norm}(s \rightarrow q)$ of flat transitions $f\left(u_{1}, \ldots, u_{n}\right) \rightarrow q^{\prime}$ where $u_{1}, \ldots, u_{n}$, and $q^{\prime}$ are states, by abstracting subterms $s^{\prime} \notin \mathcal{Q}$ of $s$ by new states. We first define the abstraction function as follows:

Definition 1. Let $\mathcal{F}$ be a set of symbols, and $\mathcal{Q}$ a set of states. For a given configuration $s \in \mathcal{T}(\mathcal{F} \cup \mathcal{Q}) \backslash \mathcal{Q}$, an abstraction of $s$ is a surjective mapping $\alpha$ :

$$
\alpha:\left\{\left.s\right|_{p} \mid p \in \operatorname{Pos}_{\mathcal{F}}(s)\right\} \mapsto \mathcal{Q}
$$

The mapping $\alpha$ is extended on $\mathcal{T}(\mathcal{F} \cup \mathcal{Q})$ by defining $\alpha$ as identity on $\mathcal{Q}$.

Definition 2. Let $\mathcal{F}$ be a set of symbols, $\mathcal{Q}$ a set of states, $s \rightarrow q$ a transition s.t. $s \in \mathcal{T}(\mathcal{F} \cup \mathcal{Q})$ and $q \in \mathcal{Q}$, and $\alpha$ an abstraction of $s$. The set $\operatorname{Norm}_{\alpha}(s \rightarrow q)$ of normalised transitions is inductively defined by: 
1. if $s=q$, then $\operatorname{Norm}_{\alpha}(s \rightarrow q)=\emptyset$, and

2. if $s \in \mathcal{Q}$ and $s \neq q$, then $\operatorname{Norm}_{\alpha}(s \rightarrow q)=\{s \rightarrow q\}$, and

3. if $s=f\left(t_{1}, \ldots, t_{n}\right)$, then $\operatorname{Norm}_{\alpha}(s \rightarrow q)=\left\{f\left(\alpha\left(t_{1}\right), \ldots, \alpha\left(t_{n}\right)\right) \rightarrow q\right\} \cup$ $\bigcup_{i=1}^{n} \operatorname{Norm}_{\alpha}\left(t_{i} \rightarrow \alpha\left(t_{i}\right)\right)$.

Example 3. Let $\mathcal{F}=\{f, g, a\}$ and $A=\left\langle\mathcal{F}, \mathcal{Q}, \mathcal{Q}_{f}, \Delta\right\rangle$, where $\mathcal{Q}=\left\{q_{0}, q_{1}, q_{2}, q_{3}\right.$, $\left.q_{4}\right\}, \mathcal{Q}_{f}=\left\{q_{0}\right\}$, and $\Delta=\left\{f\left(q_{1}\right) \rightarrow q_{0}, g\left(q_{1}, q_{1}\right) \rightarrow q_{1}, a \rightarrow q_{1}\right\}$.

- The languages recognised by $q_{1}$ and $q_{0}$ are the following: $\mathcal{L}\left(A, q_{1}\right)=$ $\mathcal{T}(\{g, a\})$, and $\mathcal{L}\left(A, q_{0}\right)=\mathcal{L}(A)=\left\{f(x) \mid x \in \mathcal{L}\left(A, q_{1}\right)\right\}$.

- Let $s=f\left(g\left(q_{1}, f(a)\right)\right)$, and $\alpha_{1}$ be an abstraction of $s$, mapping any subterm $\left.s\right|_{p}$ with $p \in \mathcal{P}_{\mathcal{P}}(s)$, to distinct states in $\left\{q_{2}, q_{3}, q_{4}\right\}$. A possible normalisation of transition $f\left(g\left(q_{1}, f(a)\right)\right) \rightarrow q_{0}$ with abstraction $\alpha_{1}$ is the following: $\operatorname{Norm}_{\alpha_{1}}\left(f\left(g\left(q_{1}, f(a)\right)\right) \rightarrow q_{0}\right)=\left\{f\left(q_{2}\right) \rightarrow q_{0}, g\left(q_{1}, q_{3}\right) \rightarrow q_{2}, f\left(q_{4}\right) \rightarrow q_{3}, a \rightarrow\right.$ $\left.q_{4}\right\}$.

\section{Applications of $\mathcal{R}^{*}(E)$ and $\mathcal{R}^{!}(E)$}

In this section we present three applications of the set of descendants and the set of normal forms to program verification.

\subsection{Sufficient Completeness}

This property has already been much investigated [3,19,23,17], in the context of algebraic specifications. We give here a definition of sufficient completeness of a TRS on a subset of the set of ground terms $E \subseteq \mathcal{T}(\mathcal{F})$.

Definition 4. A TRS $\mathcal{R}$ is sufficiently complete on $E \subseteq \mathcal{T}(\mathcal{F})$ if $\forall s \in E, \exists t \in$ $\mathcal{T}(\mathcal{C})$ s.t. $s \rightarrow_{\mathcal{R}}^{*} t$, where $\mathcal{C}$ is the set of constructors in $\mathcal{F}$.

Usual methods for checking this property on algebraic specifications are either based on enumeration and testing techniques $[19,23,17]$ or on disunification [3]. We propose, here, to check this property thanks to the set $\mathcal{R}^{!}(E)$.

Proposition 5. If the TRS $\mathcal{R}$ is $W N$ on $E \subseteq \mathcal{T}(\mathcal{F})$, and $\mathcal{R}^{!}(E) \subseteq \mathcal{T}(\mathcal{C})$, then $\mathcal{R}$ is sufficiently complete on $E$.

This comes from the fact that since $\mathcal{R}$ is WN on $E$, for all terms $s \in E, \exists t \in$ $\operatorname{IRR}(\mathcal{R})$ s.t. $s \rightarrow_{\mathcal{R}}^{*} t$. Moreover, $t \in \mathcal{R}^{!}(E)$. Since $\mathcal{R}^{!}(E) \subseteq \mathcal{T}(\mathcal{C})$, we have $t \in \mathcal{T}(\mathcal{C})$.

Example 6. Let $\mathcal{R}=\{\operatorname{app}(n i l, x) \rightarrow x, \operatorname{app}(\operatorname{cons}(x, y), z) \rightarrow \operatorname{cons}(x, \operatorname{app}(y, z))\}$, $\mathcal{F}=\mathcal{D} \cup \mathcal{C}$, where $\mathcal{D}=\{$ app $\}$ and $\mathcal{C}=\{$ cons, nil, $a\}$, and $E=\{$ app $($ nil, nil $)$, app $(\operatorname{cons}(a, n i l), n i l), \ldots$, app $(n i l$, cons $(a, n i l)), \ldots\}$. In this context, $\mathcal{R}^{!}(E)=$ $\{$ nil, cons $(a, n i l)$, cons $(a$, cons $(a, n i l)), \ldots\}$. Since $\mathcal{R}$ is terminating, $\mathcal{R}$ is WN on $E$ and since $\mathcal{R}^{!}(E) \subseteq \mathcal{T}(\mathcal{C})$, then $\mathcal{R}$ is sufficiently complete on $E$.

On the other hand, sufficient completeness on $E$ does not necessarily imply that $\mathcal{R}^{!}(E) \subseteq \mathcal{T}(\mathcal{C})$. For example, let $\mathcal{R}=\{f(a) \rightarrow a, f(a) \rightarrow f(b)\}, \mathcal{C}=\{a, b\}$ and let $E=\{f(a)\}$. Then $\mathcal{R}$ is sufficiently complete on $E$, since $f(a) \rightarrow_{\mathcal{R}} a$, but $\mathcal{R}^{!}(E)=\{a, f(b)\} \nsubseteq \mathcal{T}(\mathcal{C})$. 


\subsection{Reachability Testing}

Reachability testing consists in verifying if a term, or a term containing a pattern, can be reached by rewriting from an initial set $E$.

Definition 7. Let $\mathcal{R}$ be a TRS, $E \subseteq \mathcal{T}(\mathcal{F})$ and $t \in \mathcal{T}(\mathcal{F}, \mathcal{X})$. The pattern $t$ is $\mathcal{R}$-reachable from $E$ if there exists a ground context $C[]$, a term $s \in E$, and a substitution $\sigma$ s.t. $s \rightarrow_{\mathcal{R}}^{*} C[t \sigma]$.

It is clear that

Proposition 8. A pattern $t$ is $\mathcal{R}$-reachable from $E$ if an instance of $t$ is a subterm of an element of $\mathcal{R}^{*}(E)$.

Let us now show what can be the use of reachability testing on a simple example.

Example 9. Assume that we want to compute $A_{n}^{p}=\frac{n !}{(n-p) !}$ with the following TRS:

$$
\mathcal{R}=\left\{\begin{array}{ll}
A(n, p) \rightarrow \text { fact }(n) / f a c t(n-p) & 0 * x \rightarrow x \\
f a c t(0) \rightarrow s(0) & s(x) * y \rightarrow(x * y)+y \\
\text { fact }(s(x)) \rightarrow s(x) * \text { fact }(x) & 0 / s(y) \rightarrow 0 \\
x-0 \rightarrow x & s(x) / s(y) \rightarrow s((x-y) / s(y)) \\
0-x \rightarrow 0 & x+0 \rightarrow x \\
s(x)-s(y) \rightarrow x-y & x+s(y) \rightarrow s(x+y)
\end{array}\right\}
$$

on the domain $E=\{A(n, p) \mid n, p \in N a t\}$, where $N a t=\{0, s(0), \ldots\}$. Verifying if a division by 0 can occur is equivalent to check whether the pattern $\operatorname{div}(x, 0)$ is $\mathcal{R}$-reachable from $E$, i.e. whether $\exists C[], \exists \sigma$, s.t. $C[\operatorname{div}(x, 0) \sigma] \in \mathcal{R}^{*}(E)$.

\subsection{Termination under Sequential Reduction Strategy}

Many works are devoted to automatising termination proofs of TRSs $[1,14]$. On the other hand, it is interesting to study weaker forms of termination, since for many purposes weak normalisation is enough. In theorem provers and programming languages, rules are always applied under a specific strategy, and it is enough to ensure termination under this strategy. In addition, proving termination or WN on $\mathcal{T}(\mathcal{F}, \mathcal{X})$ or on $\mathcal{T}(\mathcal{F})$ is not always needed. In practice, a TRS is often designed to rewrite terms from a subset $E \subseteq \mathcal{T}(\mathcal{F})$, for example logical formulas in disjunctive normal form, flattened lists, or well-typed terms. Moreover, some TRSs are WN on $E \subset \mathcal{T}(\mathcal{F})$, but not on $\mathcal{T}(\mathcal{F})$ [13].

The strategy studied here is called the Sequential Reduction Strategy (SRS for short) and consists in separating a TRS $\mathcal{R}$ into several TRSs $\mathcal{R}_{1}, \ldots, \mathcal{R}_{n}$ s.t. $\mathcal{R}=\mathcal{R}_{1} \cup \ldots \cup \mathcal{R}_{n}$ and in normalising terms successively w.r.t. $\mathcal{R}_{1}, \ldots, \mathcal{R}_{n}$. This rewriting relation under SRS is denoted by $\rightarrow_{\mathcal{R}_{1} ; \ldots ; \mathcal{R}_{n}}$, and is based on modular reduction relation $[20]$.

Definition 10. Let $\mathcal{R}=\mathcal{R}_{1} \cup \ldots \cup \mathcal{R}_{n}$ be TRSs. For $s, t \in \mathcal{T}(\mathcal{F}, \mathcal{X}), s \rightarrow_{\mathcal{R}_{1} ; \ldots ; \mathcal{R}_{n}} t$ if $s$ is reducible by $\mathcal{R}$ and $\exists s_{1}, \ldots, s_{n-1} \in \mathcal{T}(\mathcal{F}, \mathcal{X})$ s.t. $s \rightarrow_{\mathcal{R}_{1}}^{!} s_{1}$ and $s_{1} \rightarrow_{\mathcal{R}_{2}}^{\prime} s_{2}$ and $\ldots$ and $s_{n-1} \rightarrow !$ 
This kind of strategy is of great interest when normalising terms w.r.t. a TRS splitted into several hierarchical TRSs (or modules) $\mathcal{R}_{1}, \ldots, \mathcal{R}_{n}$. In this situation, interleaving of rewriting steps w.r.t. to $\mathcal{R}_{1}, \ldots, \mathcal{R}_{n}$ is often not needed. If modules $\mathcal{R}_{1}, \ldots, \mathcal{R}_{n}$ are WN and share only constructors, then $\rightarrow_{\mathcal{R}_{1} ; \ldots ; \mathcal{R}_{n}}$ is terminating [13], as a corollary of results of [21,24]. Now let us give an intuition on how to prove termination of $\rightarrow_{\mathcal{R}_{1} ; \ldots ; \mathcal{R}_{n}}$ for WN TRSs $\mathcal{R}_{1}, \ldots, \mathcal{R}_{n}$ sharing function symbols. Let $\mathcal{R}=\mathcal{R}_{1} \cup \mathcal{R}_{2}$, for example. For proving termination of $\rightarrow_{\mathcal{R}_{1} ; \mathcal{R}_{2}}$ on a set of initial terms $E \subseteq \mathcal{T}(\mathcal{F})$, we need to prove that for any term $s \in E$, there is no possible infinite derivation $s \rightarrow_{\mathcal{R}_{1}}^{!} s_{1}^{\prime} \rightarrow_{\mathcal{R}_{2}}^{!} s_{1} \rightarrow_{\mathcal{R}_{1}}^{!} s_{2}^{\prime} \rightarrow_{\mathcal{R}_{2}}^{!}$ $s_{2} \rightarrow \mathcal{R}_{1} \ldots$ In that case, a criterion for proving termination of $\rightarrow_{\mathcal{R}_{1} ; \mathcal{R}_{2}}$ on $E$ is the following: construct the sets $G_{1}=\mathcal{R}_{2}^{!}\left(\mathcal{R}_{1}^{!}(E)\right), G_{2}=\mathcal{R}_{2}^{!}\left(\mathcal{R}_{1}^{!}\left(G_{1}\right)\right), \ldots$ until we get a fixpoint $G_{m}$ s.t. $G_{m}=\mathcal{R}_{2}^{!}\left(\mathcal{R}_{1}^{!}\left(G_{m}\right)\right)$. Then if $\mathcal{R}_{1}$ WN on $E, G_{1}, G_{2}, \ldots$, and $\mathcal{R}_{2}$ WN on $\mathcal{R}_{1}^{!}(E), \mathcal{R}_{1}^{!}\left(G_{1}\right), \ldots$ and if $G_{m} \subseteq I R R\left(\mathcal{R}_{1} \cup \mathcal{R}_{2}\right)$, then $\mathcal{R}$ is WN on $E$ and $\mathcal{R}$ is terminating on $E$ under SRS.

Proposition 11. If $\mathcal{R}_{1}, \ldots, \mathcal{R}_{n}$ are $W N$ resp. on subsets $E_{1}, \ldots, E_{n}$ of $\mathcal{T}(\mathcal{F})$, the rewriting relation under $S R S \rightarrow_{\mathcal{R}_{1} ; \ldots ; \mathcal{R}_{n}}$ is terminating on $E$ if the iterated sequence of sets $G_{k+1}=\mathcal{R}_{n}^{!}\left(\ldots \mathcal{R}_{1}^{!}\left(G_{k}\right) \ldots\right)$, starting from $G_{0}=E$, has a fixpoint which is a subset of $\operatorname{IRR}\left(\mathcal{R}_{1} \cup \ldots \cup \mathcal{R}_{n}\right)$, and for all $k \geq 0, G_{k} \subseteq$ $E_{1}, \mathcal{R}_{1}^{!}\left(G_{k}\right) \subseteq E_{2}, \ldots$, and $\mathcal{R}_{n-1}^{!}\left(\ldots \mathcal{R}_{1}^{!}\left(G_{k}\right) \ldots\right) \subseteq E_{n}$

\section{Approximating $\mathcal{R}^{*}(E)$ and $\mathcal{R}^{!}(E)$}

First, recall that $\mathcal{R}^{!}(E)=\mathcal{R}^{*}(E) \cap I R R(\mathcal{R}) . \operatorname{IRR}(\mathcal{R})$ is a regular tree language if $\mathcal{R}$ is left-linear [10], and a procedure for building a regular tree grammar (resp. a tree automaton) producing (resp. recognising) $I R R(\mathcal{R})$ can be found in [5]. However, $\mathcal{R}^{*}(E)$ is not necessarily a regular tree language, even if $E$ is. The language $\mathcal{R}^{*}(E)$ is regular if $E$ is regular and if $\mathcal{R}$ is either a ground TRS [7], a right-linear and monadic TRS [25], a linear and semi-monadic TRS [6] or an "inversely-growing" TRS [16], where "inversely-growing" means that every righthand side is either a variable, or a term $f\left(t_{1}, \ldots, t_{n}\right)$ where $f \in \mathcal{F}, \operatorname{ar}(f)=n$, and $\forall i=1, \ldots, n, t_{i}$ is a variable, a ground term, or a term whose variables do not occur in the left-hand side. On the other hand, for a given regular language $E, \mathcal{R}^{*}(E)$ is not necessarily regular, even if $\mathcal{R}$ is a confluent and terminating linear TRS [15]. If $\mathcal{R}$ is not "inversely-growing", then $\mathcal{R}^{*}(E)$ is not necessarily regular $[16]$.

Since our purpose is to deal with TRSs representing programs, we cannot stick to the decidable class of "inversely-growing" TRSs which is not expressive enough. Our goal here is to define, an approximation of $\mathcal{R}^{*}(E)$ i.e. a regular superset of $\mathcal{R}^{*}(E)$ for left-linear TRSs and regular sets $E$. Then, since regular langages are closed by intersection, the intersection between the regular superset of $\mathcal{R}^{*}(E)$ and $\operatorname{IRR}(\mathcal{R})$ gives a regular superset of $\mathcal{R}^{!}(E)$. Before going into details of the construction of the approximation itself, let us first show why an approximation is sufficient for proving properties addressed in Section 2. Let $\mathcal{R}$ be a TRS, and $E \subseteq \mathcal{T}(\mathcal{F})$. For any set $G$, let super $(G)$ be a regular superset of $G$. For sufficient completeness: if $\operatorname{super}\left(\mathcal{R}^{!}(E)\right) \subseteq \mathcal{T}(\mathcal{C})$ then $\mathcal{R}^{!}(E) \subseteq \mathcal{T}(\mathcal{C})$. 
For reachability testing: if $C[t \sigma] \notin \operatorname{super}\left(\mathcal{R}^{*}(E)\right)$ then $C[t \sigma] \notin \mathcal{R}^{*}(E)$. For termination under SRS: if $\operatorname{super}\left(\mathcal{R}_{n}^{!}\left(\ldots \operatorname{super}\left(\mathcal{R}_{1}^{!}\left(G_{k}\right)\right) \ldots \ldots\right)\right)$ is a subset of $\operatorname{IRR}\left(\mathcal{R}_{1} \cup \ldots \cup \mathcal{R}_{n}\right)$ then so is $\mathcal{R}_{n}^{!}\left(\ldots \mathcal{R}_{1}^{!}\left(G_{k}\right) \ldots\right)$.

Now, starting from a tree automaton $A$ s.t. $\mathcal{L}(A)=E$ and a left-linear TRS $\mathcal{R}$, we show how to build a tree automaton $\mathcal{T}_{\mathcal{R}} \uparrow(A)$ s.t. $\mathcal{L}\left(\mathcal{T}_{\mathcal{R}} \uparrow(A)\right) \supseteq \mathcal{R}^{*}(\mathcal{L}(A))$. The next proposition gives a sufficient condition for an automaton $B$ to have such a property.

Proposition 12. Let $\mathcal{R}$ be a left-linear TRS, $A=\left\langle\mathcal{F}, \mathcal{Q}, \mathcal{Q}_{f}, \Delta\right\rangle$, and $B=$ $\left\langle\mathcal{F}, \mathcal{Q}^{\prime}, \mathcal{Q}_{f}, \Delta^{\prime}\right\rangle$ two tree automata. $\mathcal{R}^{*}(\mathcal{L}(A)) \subseteq \mathcal{L}(B)$ if

1. $\Delta \subseteq \Delta^{\prime}$, and

2. $\forall l \stackrel{\rightarrow}{\rightarrow} r \in \mathcal{R}, \forall q \in \mathcal{Q}^{\prime}, \forall \sigma \in \Sigma\left(\mathcal{Q}^{\prime}, \mathcal{X}\right), l \sigma \rightarrow_{\Delta^{\prime}}^{*} q$ implies $r \sigma \rightarrow_{\Delta^{\prime}}^{*} q$.

Proof. (sketch) By definition, any term $t$ of $\mathcal{R}^{*}(\mathcal{L}(A))$ is such that $\exists s \in \mathcal{L}(A)$ s.t. $s \rightarrow_{\mathcal{R}}^{*} t$. By induction on the size of the derivation $s \rightarrow_{\mathcal{R}}^{*} t$, we prove that if $s \rightarrow_{\mathcal{R}}^{*} t$ and $s \rightarrow_{\Delta^{\prime}}^{*} q$ with $q \in \mathcal{Q}_{f}$ then $t \rightarrow_{\Delta^{\prime}}^{*} q$, which implies that $t \in \mathcal{L}(B)$. See [12] for a detailed proof.

For building $\mathcal{T}_{\mathcal{R}} \uparrow(A)$, the algorithm we propose starts from the tree automaton $A$ and incrementally adds to $\Delta$ the transitions necessary to ensure Condition 2 of Proposition 12, by computing critical peaks between rules of $\mathcal{R}$ and rules of $\Delta$ : $r \sigma \mathcal{R}^{\leftarrow} l \sigma \rightarrow_{\Delta}^{*} q$. If $r \sigma \not \rightarrow_{\Delta}^{*} q$, then it is necessary to add the transition $r \sigma \rightarrow q$ to $\Delta$. If the transition $r \sigma \rightarrow q$ is not normalised, then it has to be normalised according to Definition 2. The choice of new states used to normalise $r \sigma \rightarrow q$ is guided by the approximation function $\gamma$ defined below:

Definition 13. Let $\mathcal{Q}$ be a set of states, $\mathcal{Q}_{\text {new }}$ be a set of new states s.t. $\mathcal{Q} \cap \mathcal{Q}_{\text {new }}=\emptyset$, and $\mathcal{Q}_{\text {new }}^{*}$ the set of sequences $q_{1} \cdots q_{k}$ of states in $\mathcal{Q}_{\text {new }}$. An approximation function is a mapping $\gamma: \mathcal{R} \times\left(\mathcal{Q} \cup \mathcal{Q}_{\text {new }}\right) \times \Sigma\left(\mathcal{Q} \cup \mathcal{Q}_{\text {new }}, \mathcal{X}\right) \mapsto \mathcal{Q}_{\text {new }}^{*}$, such that $\gamma(l \rightarrow r, q, \sigma)=q_{1} \cdots q_{k}$, where $k=\mathcal{C} \operatorname{ard}\left(\mathcal{P o s}_{\mathcal{F}}(r)\right)$.

In the following, for any sequence $S=q_{1} \cdots q_{k} \in \mathcal{Q}_{\text {new }}^{*}$, and for all $i$ s.t. $1 \leq i \leq$ $k, \pi_{i}(S)$ denotes the $i$-th element of the sequence $S$, i.e. $q_{i}$.

Definition 14. (Approximation Automaton) Let $A=\left\langle\mathcal{F}, \mathcal{Q}, \mathcal{Q}_{f}, \Delta\right\rangle$ be a tree automaton, $\mathcal{R}$ a left-linear TRS, $\mathcal{Q}_{\text {new }}$ a set of new states s.t. $\mathcal{Q} \cap \mathcal{Q}_{\text {new }}=\emptyset$, and $\gamma$ an approximation function. An approximation automaton $\mathcal{T}_{\mathcal{R}} \uparrow(A)$ is a tree automaton $\left\langle\mathcal{F}, \mathcal{Q}^{\prime}, \mathcal{Q}_{f}, \Delta^{\prime}\right\rangle$ s.t.

(1) $\mathcal{Q}^{\prime}=\mathcal{Q} \cup \mathcal{Q}_{\text {new }}$, and

(2) $\Delta \subseteq \Delta^{\prime}$, and

(3) $\forall l \rightarrow r \in \mathcal{R}, \forall q \in \mathcal{Q}^{\prime}, \forall \sigma \in \Sigma\left(\mathcal{Q}^{\prime}, \mathcal{X}\right), l \sigma \rightarrow_{\Delta^{\prime}}^{*} q$ implies

$$
\operatorname{Norm}_{\alpha}(r \sigma \rightarrow q) \subseteq \Delta^{\prime}
$$

where $\alpha$ is the abstraction of $r \sigma$ defined by: $\alpha\left(\left.r \sigma\right|_{p_{i}}\right)=\pi_{i}(\gamma(l \rightarrow r, q, \sigma))$, for all $p_{i} \in \operatorname{Pos}_{\mathcal{F}}(r)=\left\{p_{1}, \ldots, p_{k}\right\}$, s.t. $p_{i} \prec p_{i+1}$ for $i=1 \ldots k-1$ (where $\prec$ is the lexicographic ordering).

By choosing specific approximation functions $\gamma$, we obtain specific approximations. 
Theorem 15. Given a tree automaton $A$ and a left-linear TRS $\mathcal{R}$, every approximation automaton satisfies: for any approximation function $\gamma$,

$$
\mathcal{L}\left(\mathcal{T}_{\mathcal{R}} \uparrow(A)\right) \supseteq \mathcal{R}^{*}(\mathcal{L}(A))
$$

Proof. (sketch) For proving $\mathcal{L}\left(\mathcal{T}_{\mathcal{R}} \uparrow(A)\right) \supseteq \mathcal{R}^{*} \mathcal{L}(A)$, it is enough to prove that the approximation automata verifies Conditions 1 and 2 of Proposition 12, for all approximation functions $\gamma$. By Definition 14, $\mathcal{T}_{\mathcal{R}} \uparrow(A)$ trivially verifies Condition 1. Then, to prove that $\mathcal{T}_{\mathcal{R}} \uparrow(A)$ also verifies Condition 2 of Proposition 12, it is enough to prove that $\operatorname{Norm}_{\alpha}(r \sigma \rightarrow q) \subseteq \Delta^{\prime}$ implies $r \sigma \rightarrow_{\Delta^{\prime}}^{*} q$. See [12] for a detailed proof.

For any rule $l \rightarrow r \in \mathcal{R}$, in order to find a $\mathcal{Q}$-substitution $\sigma$ and a state $q \in \mathcal{Q}$ s.t. $l \sigma \rightarrow_{\Delta}^{*} q$, it is possible to enumerate every possible combination of $\sigma$ and $q$ and check whether $l \sigma \rightarrow{ }_{\Delta}^{*} q$. However, this solution is not usable in practice, especially when $\mathcal{Q}$ is a large set, due to the huge number of possible $\sigma$ and $q$ to consider. In [13,12], we detail a matching algorithm which starts from a matching problem $l \unlhd q$ and a set of transitions $\Delta$, and gives every solution $\sigma: \mathcal{X} \mapsto \mathcal{Q}$ s.t. $l \sigma \rightarrow_{\Delta}^{*} q$. This algorithm is used in our implementation.

However, adding transitions to $\Delta$ may not terminate, depending on the approximation function $\gamma$ used, as in the following example.

Example 16. Let $A$ be an automaton s.t. $\Delta=\left\{\operatorname{app}\left(q_{0}, q_{0}\right) \rightarrow q_{1}, \operatorname{cons}\left(q_{2}, q_{1}\right) \rightarrow\right.$ $q_{0}$, nil $\rightarrow q_{0}$, nil $\left.\rightarrow q_{1}, a \rightarrow q_{2}\right\}, r l=\operatorname{app}(\operatorname{cons}(x, y), z) \rightarrow \operatorname{cons}(x, \operatorname{app}(y, z))$, $\mathcal{R}=\{r l\}$, and let $\gamma$ be the approximation function mapping every tuple $(r l, q, \sigma)$ to one new state $\left(\right.$ since $\mathcal{C}$ ard $\left.\left(\mathcal{P o s}_{\mathcal{F}}(\operatorname{cons}(x, \operatorname{app}(y, z)))\right)=1\right)$.

Step 1 If we apply the matching algorithm on $\operatorname{app}(\operatorname{cons}(x, y), z) \unlhd q_{1}$, we obtain a solution $\sigma=\left\{x \mapsto q_{2}, y \mapsto q_{1}, z \rightarrow q_{0}\right\}$, corresponding to the following critical peak: cons $\left(q_{2}, \operatorname{app}\left(q_{1}, q_{0}\right)\right) \mathcal{R}_{\mathcal{R}} \leftarrow \operatorname{app}\left(\operatorname{cons}\left(q_{2}, q_{1}\right), q_{0}\right) \rightarrow_{\Delta}^{*} q_{1}$. Thus, the transition to be added is $\operatorname{cons}\left(q_{2}, \operatorname{app}\left(q_{1}, q_{0}\right)\right) \rightarrow q_{1}$. Let $q_{3}$ be the new state s.t. $\gamma\left(r l, q_{1}, \sigma\right)=q_{3}$. Then, since $\mathcal{P o s}_{\mathcal{F}}\left(\operatorname{cons}\left(q_{2}, \operatorname{app}\left(q_{1}, q_{0}\right)\right)=\left\{p_{1}\right\}=\{2\}\right.$, we have $\alpha\left(\operatorname{app}\left(q_{1}, q_{0}\right)\right)=\pi_{1}\left(\gamma\left(r l, q_{1}, \sigma\right)\right)=q_{3}$, and the set of normalised transitions to be added to $\Delta$ is:

$\operatorname{Norm}_{\alpha}\left(\operatorname{cons}\left(q_{2}, \operatorname{app}\left(q_{1}, q_{0}\right)\right) \rightarrow q_{1}\right)=\left\{\operatorname{cons}\left(q_{2}, q_{3}\right) \rightarrow q_{1}, \operatorname{app}\left(q_{1}, q_{0}\right) \rightarrow q_{3}\right\}$.

Step 2 Applying the matching algorithm on $\operatorname{app}(\operatorname{cons}(x, y), z) \unlhd q_{3}$ gives a solution $\sigma^{\prime}=\left\{x \mapsto q_{2}, y \mapsto q_{3}, z \mapsto q_{0}\right\}$, corresponding to the following critical peak: $\operatorname{cons}\left(q_{2}, \operatorname{app}\left(q_{3}, q_{0}\right)\right) \mathcal{R}_{\mathcal{R}} \leftarrow \operatorname{app}\left(\operatorname{cons}\left(q_{2}, q_{3}\right), q_{0}\right) \rightarrow_{\Delta}^{*} q_{3}$. Thus, the transition to be added is $\operatorname{cons}\left(q_{2}, \operatorname{app}\left(q_{3}, q_{0}\right)\right) \rightarrow q_{3}$. Let $q_{4}$ be the new state s.t. $\gamma\left(r l, q_{3}, \sigma^{\prime}\right)=q_{4}$. Then, $\alpha\left(a p p\left(q_{3}, q_{0}\right)\right)=q_{4}$, and the set of normalised transitions to be added to $\Delta$ is:

$\operatorname{Norm}_{\alpha}\left(\operatorname{cons}\left(q_{2}, \operatorname{app}\left(q_{3}, q_{0}\right)\right) \rightarrow q_{3}\right)=\left\{\operatorname{cons}\left(q_{2}, q_{4}\right) \rightarrow q_{1}, \operatorname{app}\left(q_{3}, q_{0}\right) \rightarrow q_{4}\right\}$.

This process can go on forever and add infinitely many new states. This is due to the fact that we can apply recursively the rule $\operatorname{app}(\operatorname{cons}(x, y), z) \rightarrow$ $\operatorname{cons}(x, \operatorname{app}(y, z))$ onto infinitely growing terms recognised by the automaton $A$ (with transitions $\Delta$ ), as shown on the following figure. 


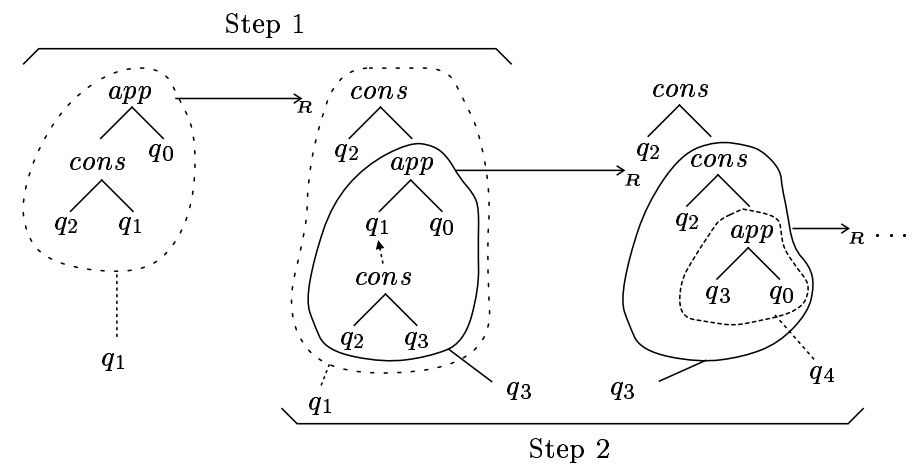

In order to have a finite automaton approximating the set $\mathcal{R}^{*}(\mathcal{L}(A))$, the intuition is to fold recursive calls into a unique new state. In the previous example, during Step 1, by applying the rule of $\operatorname{app}(\operatorname{cons}(x, y), z) \rightarrow \operatorname{cons}(x, a p p(y, z))$ on $\operatorname{app}\left(\operatorname{cons}\left(q_{2}, q_{1}\right), q_{0}\right)$, we have obtained the configuration $\operatorname{cons}\left(q_{2}, \operatorname{app}\left(q_{1}, q_{0}\right)\right)$, and we have created a new state $q_{3}$ recognising the subterm $\operatorname{app}\left(q_{1}, q_{0}\right)$. During Step 2 we have applied the same rule on the subterm $\operatorname{app}\left(q_{1}, q_{0}\right)$ recognised by $q_{3}$. In order to fold this recursive call in Step 2, we simply re-use the state $q_{3}$, instead of creating a new state $q_{4}$ for normalising the transition $\operatorname{cons}\left(q_{2}, \operatorname{app}\left(q_{3}, q_{0}\right)\right) \rightarrow q_{3}$ obtained in Step 2. Thus we obtain the set of normalised transitions $\left\{\right.$ cons $\left.\left(q_{2}, q_{3}\right) \rightarrow q_{3}, a p p\left(q_{3}, q_{0}\right) \rightarrow q_{3}\right\}$ to be added to $\Delta$. No more state nor transition needs to be further added and this automaton recognises a superset of $\mathcal{R}^{*}(\mathcal{L}(A))$. This is one of the basic idea of the ancestor approximation, which is formalised below.

Informally, every state $q \in \mathcal{Q}^{\prime}=\mathcal{Q} \cup \mathcal{Q}_{\text {new }}$ has a unique ancestor $q_{a} \in \mathcal{Q}$. The ancestor of any state $q \in \mathcal{Q}$ is $q$ itself, and the ancestor of every new state $q^{\prime} \in \mathcal{Q}_{\text {new }}$ occuring in the sequence $\gamma(l \rightarrow r, q, \sigma)$ (used to normalise a new transition $r \sigma \rightarrow q$ ), is the ancestor of $q$. In the ancestor approximation, (1) the $\gamma$ function does not depend on the $\sigma$ parameter and, (2) for any new state $q^{\prime} \in \mathcal{Q}_{\text {new }}, \gamma\left(l \rightarrow r, q^{\prime}, \sigma\right)=\gamma(l \rightarrow r, q, \sigma)$, where $q \in \mathcal{Q}$ is the ancestor of $q^{\prime}$.

Definition 17. An approximation function $\gamma$ is called ancestor approximation if

1. $\forall l \rightarrow r \in \mathcal{R}, \forall q \in \mathcal{Q}^{\prime}, \forall \sigma_{1}, \sigma_{2} \in \Sigma\left(\mathcal{Q}^{\prime}, \mathcal{X}\right)$,

$$
\gamma\left(l \rightarrow r, q, \sigma_{1}\right)=\gamma\left(l \rightarrow r, q, \sigma_{2}\right), \text { and }
$$

2. $\forall l_{1} \rightarrow r_{1}, l_{2} \rightarrow r_{2} \in \mathcal{R}, \forall q \in \mathcal{Q}^{\prime}, \forall q_{1}, \ldots, q_{k} \in \mathcal{Q}_{n e w}, \sigma_{1}, \sigma_{2} \in \Sigma\left(\mathcal{Q}^{\prime}, \mathcal{X}\right)$,

$$
\gamma\left(l_{1} \rightarrow r_{1}, q, \sigma_{1}\right)=q_{1} \ldots q_{k} \Rightarrow \forall i=1 \ldots k, \gamma\left(l_{2} \rightarrow r_{2}, q_{i}, \sigma_{2}\right)=\gamma\left(l_{2} \rightarrow r_{2}, q, \sigma_{2}\right) .
$$

Note that in the particular case of Example 16, using the ancestor approximation, we have $\gamma\left(r l, q_{1}, \sigma\right)=q_{3}$, and by case 2 of Definition 17 we get $\gamma\left(r l, q_{3}, \sigma^{\prime}\right)=$ $\gamma\left(r l, q_{1}, \sigma^{\prime}\right)$, by case 1 we get that $\gamma\left(r l, q_{1}, \sigma^{\prime}\right)=\gamma\left(r l, q_{1}, \sigma\right)=q_{3}$, thus we have $\gamma\left(r l, q_{3}, \sigma^{\prime}\right)=q_{3}$, and the construction of $\mathcal{T}_{\mathcal{R}} \uparrow(A)$ becomes finite.

Theorem 18. Approximation automata built using ancestor approximation are finite automata. 
Proof. (sketch) The automaton $\mathcal{T}_{\mathcal{R}} \uparrow(A)$ is finite if the set of new states $\mathcal{Q}_{\text {new }}$ is finite. Since $\mathcal{Q}$ is finite, $\mathcal{R}$ is finite, and $\gamma$ does not depend on the $\sigma$ parameter, there is a finite number of distinct sequences $\gamma(l \rightarrow r, q, \sigma)$ for $l \rightarrow r \in \mathcal{R}$, $q \in \mathcal{Q}$, and these sequences are finite. On the other hand, every state $q^{\prime} \in$ $\mathcal{Q}_{\text {new }}$ has a unique ancestor $q \in \mathcal{Q}$, and $\gamma\left(l \rightarrow r, q^{\prime}, \sigma\right)=\gamma(l \rightarrow r, q, \sigma)$. Thus, there is a finite number of distinct sequences $\gamma\left(l \rightarrow r, q^{\prime}, \sigma\right)=q_{1}^{\prime} \ldots q_{n}^{\prime}$ with $q^{\prime}, q_{1}^{\prime}, \ldots, q_{n}^{\prime} \in \mathcal{Q}_{\text {new }}$. Hence, there is a finite number of states in $\mathcal{Q}_{\text {new }}$, used to normalise transitions. See [12] for a detailed proof.

\section{Experiments}

Working on tree automaton by hand is always a heavy task. In order to experiment and check feasibility of the method, we have implemented in ELAN [18] a library of usual algorithms on tree automaton: union, intersection, cleaning, inclusion test, as well as algorithms for building the tree automata $\mathcal{T}_{\mathcal{R}} \uparrow(A)$, and $A_{I R R(\mathcal{R})}$ (the automaton recognising the set $\operatorname{IRR}(\mathcal{R})$ ) for a given automaton $A$ and a given left-linear TRS $\mathcal{R}$. In all the following examples, we use the same ancestor approximation method. We have experimented with several other approximations: if the $\gamma$ function does not depend on the rule $l \rightarrow r$, on the state $q$ or on the position $p$, then the approximation automaton is smaller, and faster to compute. However, the recognised language is bigger and sometimes not precise enough for our purpose. On the other hand, if for every $\sigma$, the $\gamma$ function have distinct values, then the construction of the automaton is not necessarily terminating.

\subsection{Reachability Testing}

Let $\mathcal{R}_{1}$ be a TRS computing the function $A_{n}^{p}=\frac{n !}{(n-p) !}$, and $\operatorname{Aut}(0)$ a tree automaton recognising the set $\mathcal{L}(\operatorname{Aut}(0))=\mathcal{L}\left(\operatorname{Aut}(0), q_{0}\right)=\{A(n, p) \mid n, p \in$ $\left.\mathcal{L}\left(\operatorname{Aut}(0), q_{1}\right)\right\}$ where $\mathcal{L}\left(\operatorname{Aut}(0), q_{1}\right)=N a t=\{0, s(0), \ldots\}$. The TRS $\mathcal{R}_{1}$ and the automaton $A u t(0)$ are given as input to our prototype in the following syntax:
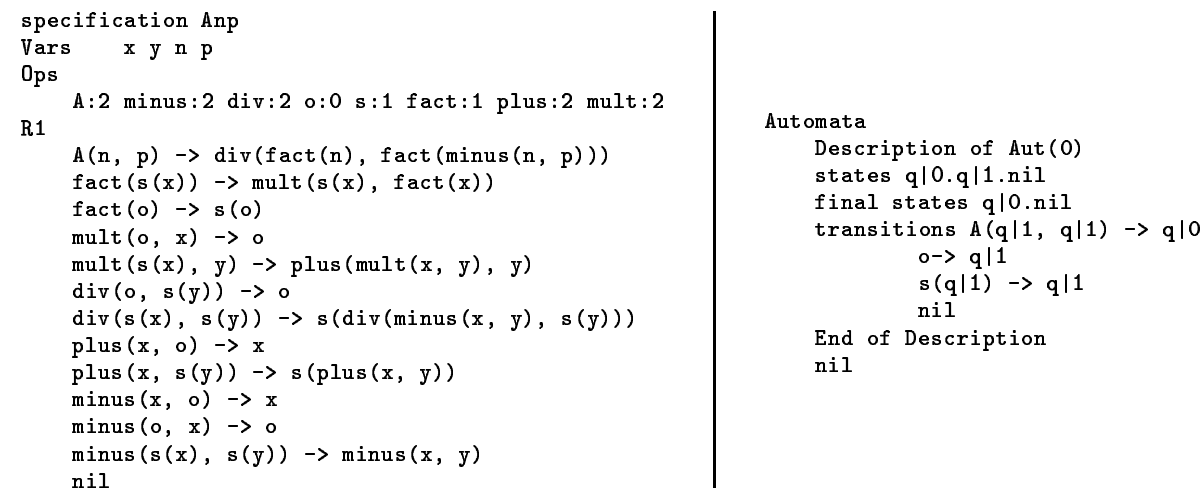

Computing the automaton $\mathcal{T}_{\mathcal{R}_{1} \uparrow}(\operatorname{Aut}(0))$ s.t. $\mathcal{L}\left(\mathcal{T}_{\mathcal{R}_{1}} \uparrow(A u t(0))\right) \supseteq \mathcal{R}_{1}^{*}(\mathcal{L}(\operatorname{Aut}(0)))$, can be achieved by evaluating the following query: T_up (R1) on (Aut (0)), and the result is the automaton $\operatorname{Aut}(1)$ : 


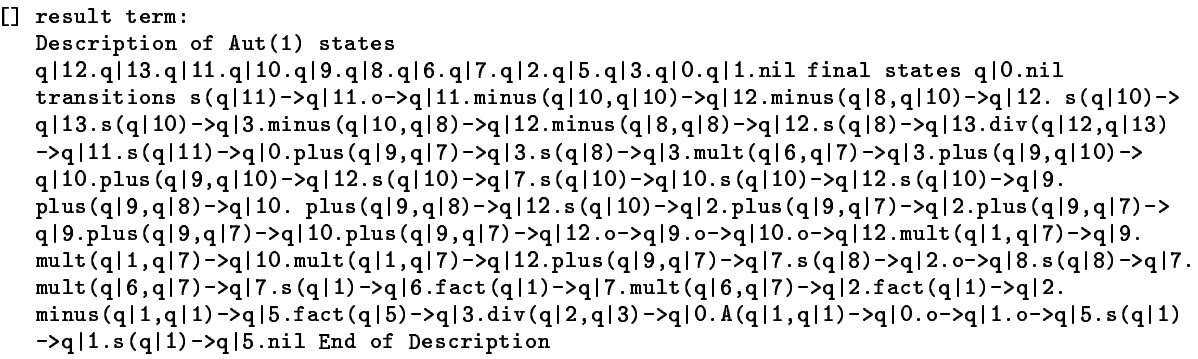

The pattern $\operatorname{div}(x, 0)$ is not $\mathcal{R}_{1}$-reachable from $\mathcal{L}(A u t(0))$ if for all ground contexts $C$ [] and all substitutions $\sigma, C[\operatorname{dix}(x, 0) \sigma] \notin \mathcal{L}\left(\mathcal{T}_{\mathcal{R}_{1}} \uparrow(A u t(0))\right)$. This can be checked evaluating the following query: (div $(\mathrm{x}, 0) ?=$ states) with (Aut (1)). The result is

[] result term: nil

meaning that there exists no substitution $\sigma$ and no state $q \in \mathcal{Q}$ s.t. $\operatorname{div}(x, 0) \sigma \rightarrow_{\Delta}^{*}$ $q$, where $\mathcal{Q}$ and $\Delta$ are respectively the set of states and the set of transitions of Aut(1). An interesting aspect of this method is that the automaton $\mathcal{T}_{\mathcal{R}} \uparrow(A)$ is computed once for all, and the check itself is a simple and low cost operation. Another advantage is that for computing $\mathcal{T}_{\mathcal{R}} \uparrow(A)$, the TRS $\mathcal{R}$ is not supposed to be terminating nor even weakly normalising. This is of great interest when using TRS to encode non-terminating systems, like systems of communicating processes, for example. Note that such non-terminating TRS cannot be handled by induction proof techniques that need a well-founded ordering for proving termination of the TRS.

\subsection{Sufficient Completeness}

In order to prove sufficient completeness of $A(n, p)$ with $n, p \in N a t$, we first compute the intersection automaton between $A u t(1)$, computed previously, and the automaton recognising the set $I R R\left(\mathcal{R}_{1}\right)$, computed by the function build_nf (R1). The query is simplify (Aut (1) inter build_nf(R1)), and the result is:

[] result term:

Description of Aut(2) states q/0.q|1.nil final states q/1.nil

transitions $s(q \mid 0)->q|1 . s(q \mid 0)->q| 0.0->q \mid 0$. nil End of Description

Thus, the superset of $\mathcal{R}_{1}^{!}(\mathcal{L}(\operatorname{Aut}(0)))$ is $\mathcal{L}(\operatorname{Aut}(2))=\mathcal{L}\left(\operatorname{Aut}(2), q_{1}\right)=\{s(x) \mid x \in$ $\left.\mathcal{L}\left(\operatorname{Aut}(2), q_{0}\right)\right\}$, and $\mathcal{L}\left(\operatorname{Aut}(2), q_{0}\right)=\{0, s(0), \ldots\}$. Thus $\mathcal{L}\left(\operatorname{Aut}(2), q_{0}\right)=N a t$, and $\mathcal{L}(\operatorname{Aut}(2))=N a t^{*}$. Therefore, we trivially have $\mathcal{R}_{1}^{!}(\mathcal{L}($ Aut $(0))) \subseteq N a t^{*} \subseteq$ $\mathcal{T}(\mathcal{C})$ and if $\mathcal{R}_{1}$ is weakly normalising on terms $A(n, p)$ with $n, p \in$ Nat, then $\mathcal{R}_{1}$ is also sufficiently complete on those terms. Note that, if $A u t(2)$ is more complex, inclusion between automaton $\operatorname{Aut}(2)$ and an automaton recognising exactly $\mathcal{T}(\mathcal{C})$ can also be verified automatically by our prototype. 
seems to be sufficient for our purposes in many practical cases. Approximation of regular language is a notion that was already used in in [16], but in a different way and for a different purpose. In [16], Jacquemard approximates a TRS by another one for which the set of descendants is regular, whereas in our approach, we approximate the set of new states used for normalising transitions, in order to fold recursion when necessary. The set of descendants can be computed exactly thanks to the Tree Tuple Synchronised Grammars (TTSG) approach of non-regular langages proposed in [22]. However, this approach deals with more restricted classes of TRSs; namely linear confluent constructor systems. Moreover, in practice, efficiency of TTSGs for our purposes is not obvious.

A promising application area is the study of non-terminating TRSs encoding the behaviour of systems of communicating processes or systems of parallel processes sharing memory. In this framework, we can prove that there is no deadlock and also some general "reachability" properties: ensure mutual exclusion, ensure that a process never stops, etc. In further research, we intend to compute another regular approximation: a subset of $\mathcal{R}^{*}(E)$ in order to achieve some reachability testing in the other way: for instance to prove that a specific behaviour must occur, we may have to check that a specific pattern does occur in the set of $\mathcal{R}$-descendants. We also would like to get rid of the left-linear limitation in order to enlarge the class of programs to be checked, and to compute more precise approximations.

\section{Acknowledgements}

I would like to thank Hélène Kirchner, Christophe Ringeissen, Aart Middeldorp, Bernhard Gramlich, Isabelle Gnaedig for comments and discussion on this work, Florent Jacquemard, Marc Tommasi, Gregory Kucherov for discussion on tree automata and regular tree langages, as well as referees for their useful comments.

\section{References}

1. T. Arts and J. Giesl. Automatically proving termination where simplification orderings fail. In M. Bidoit and M. Dauchet, editors, Proc. 22nd CAAP Conf., Lille (France), volume 1214 of LNCS, pages 261-272. Springer-Verlag, 1997.

2. T. Arts and J. Giesl. Proving innermost termination automatically. In Proc. 7th RTA Conf., Sitges (Spain), volume 1232 of LNCS, pages 157-171. Springer-Verlag, 1997.

3. H. Comon. Sufficient completeness, term rewriting system and anti-unification. In J. Siekmann, editor, Proc. 8th CADE Conf., Oxford (UK), volume 230 of LNCS, pages 128-140. Springer-Verlag, 1986.

4. H. Comon, M. Dauchet, R. Gilleron, D. Lugiez, S. Tison, and Tommasi. Tree automata techniques and applications. Preliminary Version, http://13ux02.univ-lille3.fr/tata/, 1997.

5. H. Comon and J.-L. Rémy. How to characterize the language of ground normal forms. Technical Report 676, INRIA-Lorraine, 1987.

6. J. Coquidé, M. Dauchet, R. Gilleron, and S. Vágvölgyi. Bottom-up tree pushdown automata and rewrite systems. In R. V. Book, editor, Proc. 4th RTA Conf., Como (Italy), volume 488 of $L N C S$, pages 287-298. Springer-Verlag, 1991. 
7. M. Dauchet and S. Tison. The theory of ground rewrite systems is decidable. In Proc. 5th LICS Symp., Philadelphia (Pa., USA), pages 242-248, June 1990.

8. N. Dershowitz and C. Hoot. Natural termination. TCS, 142(2):179-207, May 1995.

9. N. Dershowitz and J.-P. Jouannaud. Handbook of Theoretical Computer Science, volume B, chapter 6: Rewrite Systems, pages 244-320. Elsevier Science Publishers B. V. (North-Holland), 1990. Also as: Research report 478, LRI.

10. J. H. Gallier and R. V. Book. Reductions in tree replacement systems. TCS, $37: 123-150,1985$.

11. F. Gécseg and M. Steinby. Tree automata. Akadémiai Kiadó, Budapest, Hungary, 1984.

12. T. Genet. Decidable approximations of sets of descendants and sets of normal forms (extended version). Technical report, INRIA, 1997.

13. T. Genet. Proving termination of sequential reduction relation using tree automata. Technical report, CRIN, 1997. 97-R-091, 28 pages.

14. T. Genet and I. Gnaedig. Termination proofs using gpo ordering constraints. In M. Dauchet, editor, Proc. 22nd CAAP Conf., Lille (France), volume 1214 of LNCS, pages 249-260. Springer-Verlag, 1997.

15. R. Gilleron and S. Tison. Regular tree languages and rewrite systems. Fundamenta Informaticae, 24:157-175, 1995.

16. F. Jacquemard. Decidable approximations of term rewriting systems. In H. Ganzinger, editor, Proc. 7th RTA Conf., New Brunswick (New Jersey, USA), pages 362-376. Springer-Verlag, 1996.

17. D. Kapur, P. Narendran, and H. Zhang. On sufficient completeness and related properties of term rewriting systems. Acta Informatica, 24:395-415, 1987.

18. C. Kirchner, H. Kirchner, and M. Vittek. Designing constraint logic programming languages using computational systems. In P. Van Hentenryck and V. Saraswat, editors, Principles and Practice of Constraint Programming. The Newport Papers., chapter 8, pages 131-158. The MIT press, 1995.

19. E. Kounalis. Completeness in data type specifications. In B. Buchberger, editor, Proceedings EUROCAL Conference, Linz (Austria), volume 204 of LNCS, pages 348-362. Springer-Verlag, 1985.

20. M. Kurihara and I. Kaji. Modular term rewriting systems and the termination. IPL, 34:1-4, Feb. 1990.

21. M. Kurihara and A. Ohuchi. Modular term rewriting systems with shared constructors. Journal of Information Processing of Japan, 14(3):357-358, 1991.

22. S. Limet and P. Réty. E-unification by means of tree tuple synchronized grammars. In M. Dauchet, editor, Proc. 22nd CAAP Conf., Lille (France), volume 1214 of $L N C S$, pages 429-440. Springer-Verlag, 1997.

23. T. Nipkow and G. Weikum. A decidability result about sufficient completeness of axiomatically specified abstract data types. In 6th GI Conference, volume 145 of LNCS, pages 257-268. Springer-Verlag, 1983.

24. E. Ohlebusch. Modular Properties of Composable Term Rewriting Systems. PhD thesis, Universität Bielefeld, Bielefeld, 1994.

25. K. Salomaa. Deterministic Tree Pushdown Automata and Monadic Tree Rewriting Systems. J. of Computer and System Sciences, 37:367-394, 1988. 